\title{
Vibro-acoustic and nonlinear analysis of cadavric femoral bone impaction in cavity preparations
}

\author{
Sebastian Oberst ${ }^{1,2,3,6, \star}$, Johanna Baetz ${ }^{4}$, Graeme Campbell ${ }^{4}$, Frank Lampe ${ }^{5}$, Joseph C.S. Lai ${ }^{6}$, Norbert \\ Hoffmann ${ }^{2,3}$, and Michael Morlock ${ }^{4}$, \\ ${ }^{1}$ University of Technology Sydney, Centre for Audio, Acoustics and Vibration, Faculty of Engineering and Information Tech- \\ nology, Sydney, Australia \\ ${ }^{2}$ Hamburg University of Technology, Dynamics Group, Mechanical Engineering, Hamburg, Germany \\ ${ }^{3}$ Imperial College London, Department of Mechanical Engineering, London, United Kingdom \\ ${ }^{4}$ Hamburg University of Technology, Institute of Biomechanics, Mechanical Engineering, Hamburg, Germany \\ ${ }^{5}$ Hamburg University of Applied Sciences, Department of Life Sciences, Hamburg, Germany \\ ${ }^{6}$ University of New South Wales Canberra, School of Engineering and IT, Canberra, Australia
}

\begin{abstract}
Owing to an ageing population, the impact of unhealthy lifestyle, or simply congenital or gender specific issues (dysplasia), degenerative bone and joint disease (osteoarthritis) at the hip pose an increasing problem in many countries. Osteoarthritis is painful and causes mobility restrictions; amelioration is often only achieved by replacing the complete hip joint in a total hip arthroplasty (THA). Despite significant orthopaedic progress related to THA, the success of the surgical process relies heavily on the judgement, experience, skills and techniques used of the surgeon. One common way of implanting the stem into the femur is press fitting uncemented stem designs into a prepared cavity. By using a range of compaction broaches, which are impacted into the femur, the cavity for the implant is formed. However, the surgeon decides whether to change the size of the broach, how hard and fast it is impacted or when to stop the excavation process, merely based on acoustic, haptic or visual cues which are subjective. It is known that non-ideal cavity preparations increase the risk of peri-prosthetic fractures especially in elderly people.

This study reports on a simulated hip replacement surgery on a cadaver and the analysis of impaction forces and the microphone signals during compaction. The recorded transient signals of impaction forces and acoustic pressures $(\approx 80 \mu \mathrm{s}-2 \mathrm{~ms})$ are statistically analysed for their trend, which shows increasing heteroscedasticity in the force-pressure relationship between broach sizes.

TIKHONOV regularisation, as inverse deconvolution technique, is applied to calculate the acoustic transfer functions from the acoustic responses and their mechanical impacts. The extracted spectra highlight that system characteristics altered during the cavity preparation process: in the high-frequency range the number of resonances increased with impacts and broach size. By applying nonlinear time series analysis the system dynamics increase in complexity and demand for a larger minimum embedding dimension. The growing number of resonances with similar level of the transfer function indicates a higher propensity to dissipate energy over sound; the change in embedding dimension indicates a decrease in linearity. The spectral changes as well as the altered dimension requirements indicate either an improved coupling between the bone and the broach or the onset of micro-fractures caused by growing stress levels within the bone.
\end{abstract}

\section{Introduction}

Inflammatory bone degeneration (osteoarthritis) often requires the replacement of the whole synovial joint, i.e. total arthroplasty [1] with total hip (THA) and knee replacements (TKA) become more common as reported by the Australian National Joint Replacement Registry [2]. Metrics such as the ASA score (American Society of Anaesthesiologists - Physical Status Classification) or the Body Mass Index (BMI) [2] indicate that an longer life expectancy, increasingly unhealthy lifestyles and the growing risk of overweight result in more problems associated with joints such as the knee, the shoulder or the hip [1]. In

\footnotetext{
^e-mail: sebastian.oberst@uts.edu.au
}

2015 about 498,660 hip, and 592,577 knee joint replacement procedures $(1,123,643$ in total) were registered, an increase of $2.6 \%$ and $5.9 \%$, respectively over those in 2014 [2]. Hip prosthesis systems consist of a ball joint which is tapered to a stem component. The stem is press fitted to the femur and the ball joint runs in the liner of the acetabular cup which is inserted into the acetabulum [3]. Implant materials are hard $(\mathrm{H})$ metal alloys (steel, titanium-based or more often made of cobalt-chromium), alumina/composite ceramics or soft $(\mathrm{S})$ plastics e.g. the ultra-high molecular weight polyethylene [4]. Hard-on-hard $(\mathrm{HoH})$ bearing articulations, i.e. ceramic-on-ceramic or hard-on-soft combinations can be installed [4]. 
The cavity for the stem is prepared using the impaction of broaches and a surgical hammer; here in particular the primary stability of the artificial joint is important. Primary stability is achieved through an interference-fit which strongly depends on the bone-implant contact [5]. Preparation of the proximal femur cavity prior to implantation is achieved via compaction (bone is crushed to form the cavity) or extraction (bone is removed to form the cavity) using incremental broaching. As a result of the cavity preparation generally a higher bone densification close to the cavity region in the bone-implant envelope can be found [6]. During THA the surgeon decides when and whether to change the size of the broach, how hard and fast to impact it and when to stop the cavity preparation [7]. This decision is subjective, generally based on changes in pitch, haptic or visual cues [7]. However, owing to non-ideal cavity preparation, lysis (implant loosening) and post-operative prosthesis dislocation due to insufficient stability at the time of implantation may occur which is a major cause for revision surgeries $[2,4]$. On the other hand, by increasing the primary stability too far the risk of peri-prosthetic fractures actually grows [4, 8].

Yagi et al. [9] used an ultrasonic system within an instrumented hammer to measure the acoustic signal's attenuation time to decide on the most suitable broach and showed that the attenuation time reduced for broaches with a larger femoral canal, presumably due to increased compaction of the cancellous bone and an enhanced connection between the implant and the tissue [9]. Mathieu et al. [10] studied the variation of the impact duration during in-vitro insertions of acetabular cup implants which decreased at first as a function of the impact number, then reached a stationary value equal to about $4.2 \pm 0.7 \mathrm{~ms}$ after an average number of about 4 impacts. It was hypothesised that the impact duration is related to the bone-implant contact variations.

Sakai et al. [7] studied the relation between the hammering frequency during insertion by measuring both force and sound on a clamped femur. The internal stress during implantation is estimated using the dynamic explicit finite element solver LSDYNA. A reduction in frequency correlated well with the increased stress levels $\sigma$ which suggests that once the maximum $\sigma$ is reached the hammering force can be reduced [7].

Whitwell et al. [11] studied the spectral components of the acoustic signal during impaction for nine plant insertion surgeries which were independently conducted by two surgeons (six and three samples). For the last broach a low-frequency component at around 0.4-1.2 kHz was detected, which they attributed to the resonant frequency of a standing sound wave within the femoral canal (estimated at about $894 \mathrm{~Hz}$ ); other peaks were assumed to be transverse vibration modes of the implant components. Whitwell et al. [11] suggested the low frequency component as an indicator of the optimal broach size being reached. Morohashi et al. [12] studied the sound pressure levels of the impacting hammer of the first and the second dominant resonances in 71 total hip arthroplasties; a pattern with a smaller number accentuated resonances in the spectrum showed significantly less intraoperative fractures and subsidence.

Especially within the last five years some progress has been made in identifying acoustic features which help surgeons when to stop impacting the insertion broach. However, this progress is very limited and the findings do not have any practical use yet. From past studies important information, such as the sampling rate is missing and never the change in the system itself has been studied but only its acoustic response alone. Here we measure the radiated sound and the impaction forces during cavity preparation using compaction broaches in a cadaver THA study in the theatre. We analyse the sequence of impactions for each broach and between broaches and calculate acoustic transfer functions as unique descriptors of the system to radiate sound due to impulsive excitation, cf. [13]. Nonlinear dynamics tools and complexity measures, in particular nonlinear time series analyses (NTSA) methods have rarely been applied in biomechanics [14] although these methods could provide insights for complex nonlinear data which linear methods could not [15]. We therefore apply NTSA tools to analyse the impulse response signal in order to determine objectively the change of complexity i.e. the change of phase space dimensions required in course of the impaction process.

\section{Materials and Methods}

\subsection{Experiments}

The hip of a female 54-year-old cadaver was scanned with quantitative computer tomography (Philips Brilliance $16,1 \mathrm{~mm}$ slice thickness) including a measurement sphere for templating. An experienced surgeon determined the adequate implant size with templates and resected the femoral head using an anterolateral approach. A chisel was used to excise the residual femoral neck and a blunt reamer demonstrated the canal axis. Six stainless steel compaction broaches of increasing size were introduced to prepare the cancellous bone bed. Impaction forces from

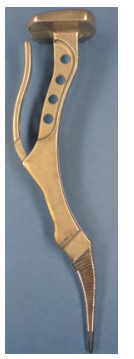

(a)

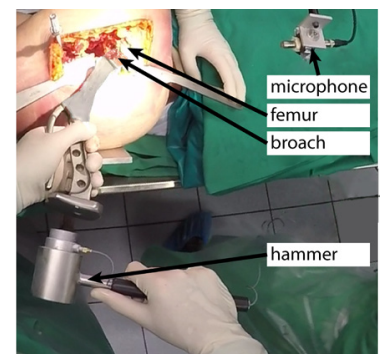

(b)
Figure 1. (a) Broach attached to the broach handle and (b) experimental setup in the operation theatre

the surgeon were recorded at the tip of an instrumented surgical hammer $(1.4 \mathrm{~kg})$ that was modified with a dynamic piezoelectric load cell (capacity $89 \mathrm{kN}, 200 \mathrm{C} 20$, PCB Piezotronics, Depew, New York, USA). The acoustic signal was recorded with an ICP microphone system 
(378B02, PCB Piezotronics, Depew, New York, USA)) within $28 \mathrm{~cm}$ distance to the calcar. For data acquisition a 16-bit module was used (NI 9222, National Instruments, Austin, TX, USA). Both signals were triggered by hammer impaction and were recorded for $3 \mathrm{~ms}$ with a sampling frequency of $500 \mathrm{kHz}$ via a Labview (National Instrument, Austin TX, USA) interface.

\subsection{Statistical analysis}

Before the data was analysed, outliers (near zero impaction force and or near zero sound pressure level, as identified visually and by box plots) were discarded. Histograms of all recorded impaction and all acoustic signals were generated. The theoretical continuous density is estimated using the EPANECHNIKOV kernel method and the probabilities of occurrence were calculated. Then the maximum value in acoustic pressure and impact force for each hit and broach were identified and plotted in a scatter plot in order to identify obvious changes between single hits or between different broaches. The maximum values of sound pressure $(\mathrm{Pa})$ are plotted against their maximum impaction force $(\mathrm{kN})$.

\subsection{Acoustic transfer function}

To determine the system characteristics, i.e. the ability of the tissue/bone-broach interface of the cadaver-implantsystem to radiate sound owing to impaction, acoustic transfer functions are determined. We therefore recall the principle of an open-loop controller, i.e. the output $(z)$ does not have any effect on the control of the input values $(x)$, bounded by the system $(g)$ considering only single hits [16]. To calculate the transfer function the spectrum of the impact as source signal and that of the microphone as receiver signal is used, similar to [17] only that now the transfer function is unknown (cf. water level method, Gubbins [18]). In Eq. (1) this relation between the input (source), the system and output (receiver) is expressed in the time domain using convolution integrals; by using the FOURIER domain $(\mathcal{F})$ the convolution changes to a multiplication; and considering a spectrum, a vector notation is used (bold letters).

$$
x(t) * g(t)=z(t) \stackrel{\mathcal{F}}{\longrightarrow} X(\omega) G(\omega)=Z(\omega) \stackrel{\vec{\cdot})}{\longrightarrow} \mathbf{X G}=\mathbf{Z}
$$

Calculating backwards, simplifying $X(\omega)=X$ and conducting an inverse FOURIER transform provides the system's impulse response function $g(t)$, of which we take the real part $\mathfrak{R}$

$$
\mathbf{X G}=\mathbf{Z} \stackrel{X^{-1}(\cdot)}{\longrightarrow} \mathbf{G}=\mathbf{X}^{-\mathbf{1}} \mathbf{Z} \stackrel{\mathcal{F}^{-1}}{\longrightarrow} \mathfrak{R}(g(t)) .
$$

However, a real-life system is often ill-conditioned with noise in the signal and even for simulation data, the application of an inverse FOURIER transformation generally is problematic [17]. Attempts of signal enhancement with deconvolution can be found in image processing with a point-spread function using wavelet-filtering with soft/hard BAYESIAN thresholding (regularisation) [19].
Another well established method is the water level method [18] or the TIKHONOV regularisation [20], which we use here by adding uncorrelated noise to the denominator to improve the matrix condition [20].

Let $\mathbf{X}$ be the Discrete Fourier Transform of the impaction signal (complex vector), $\mathbf{G}$ be the transfer function of the broach and the cadaver, and $\mathbf{Z}$ be the measured acoustic response. Minimisation of the EUCLIDEAN norm of the difference [18] $\epsilon^{2}=\|\mathbf{X} \cdot \mathbf{G}-\mathbf{Z}\|_{2}^{2}$ gives an estimate of $\mathbf{G}$, which is generally overdetermined. Due to overdetermination the so-called TIKHONOV or water-level matrix for regularisation is incorporated which leads to the following expression of the excitation signal.

$$
\mathbf{G}=\frac{\mathbf{Z} \overline{\mathbf{X}}}{\mathbf{X} \overline{\mathbf{X}}+\overline{\mathbf{\Gamma}}}
$$

with $\overline{\mathbf{G}}$ being the Hermitian of matrix $\mathbf{G}$ and $\boldsymbol{\Gamma}=$ $\sqrt{\lambda 1}$ being the regularisation matrix equal to the squareroot of the water-level control parameter $\lambda$ times a vector, which includes only entries of unity [20].

To calculate the FOURIER spectra, a sampling rate of $0.5 \mathrm{MHz}$ and a time series length of $L=1,500$ samples were selected. The choice of $\lambda$ is based on comparing spectra within a defined frequency range, here zero to 250 $\mathrm{kHz}$ [17] (for $\lambda=1.2 \times 10^{2}$ ). For each hammer hit a transfer function was attracted and plotted for each broach separately. Then, the fundamental frequencies and their peaks (i.e. the fundamental resonances) of the transfer functions were extracted using MATLAB's peak search algorithm.

\subsection{Nonlinear time series analysis}

In order to confirm a change in the characteristics of the system from being mono-frequent to multi-frequent with greater broach size, (i.e. an increase in complexity), a nonlinear time series analysis is conducted next. The transfer function is converted into its real-valued impulse response $g(t)$ (as a linear time-invariant system's response valid for all frequencies) by conducting an inverse FOURIER transformation (Eq. 2).

The changes of the system characteristics with progressing impaction were estimated by estimating a time delay and an embedding dimension of the impulse response function $g(t)$ (Eq. 2). As complexity measures, the averaged auto-mutual information [21, 22] (10 bins) and the global false nearest neighbour algorithm (maximum norm, falseness of 5 neighbours) were employed [23-25]. To study significant differences, one-way analysis of variance (ANOVA) tests were conducted and combined with a BONFERRONI correction in a multi-compare study to account for group size effects, both at a 5\% significance level; impacts belonging to one broach were assigned to one group. All analyses were conducted using MATLAB 2016 b.

\section{Results}

\subsection{Experiments}

Broaches one to six were hit 27, 22, 18, 25, 25 and 35 times. Normalised time traces of the impaction and the 
microphone signal of the second broach are exemplified in Fig. 2. No obvious difference between the different hits could be observed. After the maximum impaction force is reached, the impulse attenuates quickly and it takes about $1 \mathrm{~ms}$ until the maximum sound pressure is reached due to travel time of the sound wave to the microphone.

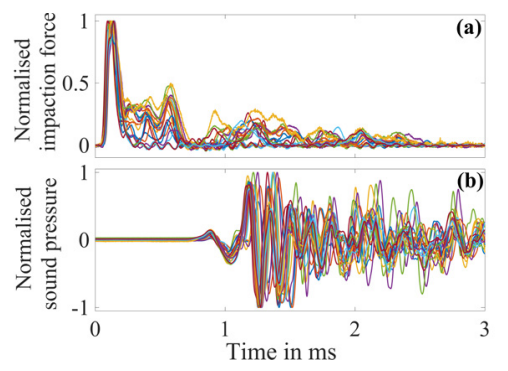

Figure 2. Normalised (a) impaction forces; and (b) sound pressures for impacting the second broach (22 measurements each in total)

\subsection{Statistical analysis}

For broaches 2, 4 and 6, one data point each with either near zero force or frequency is discarded. Distributions of all maximum impaction amplitudes and all maximum sound pressure in Fig. 3 reveal that they are positively skewed. While the impact force appears uni-modal in the range between $1 \mathrm{kN}$ and $19 \mathrm{kN}$, the sound pressure signal (1 $\mathrm{Pa}$ to $37 \mathrm{~Pa}$ ) appears to be multimodal consisting of at least two superposed GAUSSIANS. Plotting maximum

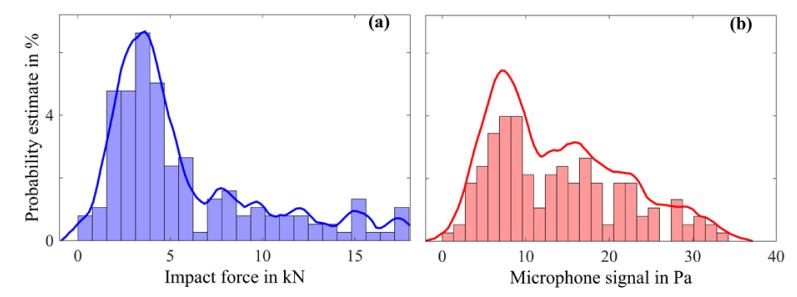

Figure 3. Statistical distribution of maximum amplitudes: impaction signal; and (b) sound pressure signal

sound pressure (dependent variable) against the maximum impaction force (independent variable) for each impact and each broach in a scatter plot (Figure 4) shows that for the first three broaches, maximum impaction forces were less than $5 \mathrm{kN}$, but that for the last three broaches, they were up to $19 \mathrm{kN}$. Overall, the data appears linearly correlated but heteroscedastic in character, i.e. an increased variability with larger broaches used. Further, for a given impaction force, the sound pressure produced for broach 4 seems to be significantly higher than all the other broaches except for low impaction force.

\subsection{Acoustic transfer function}

After varying $\lambda$ in a parametric study from $10^{-6}$ to $10^{6}$ in 101 steps it was set to $1.2 \times 10^{2}$. If $\lambda$ was chosen too

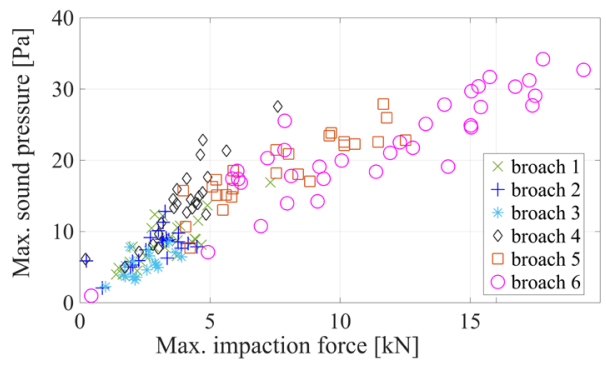

Figure 4. Scatter plot data plotted in Fig. 3 of all six broaches.

small, the transfer function (TF) experienced a low frequency distortion; for a $\lambda$ too large the amplitude of the TF decreased too quickly after having converged to the same level [17]. Families of transfer functions for broaches 1 to 6 are depicted in Figure 6. Diamond markers indicate the fundamental frequency for each hit. Dashed vertical lines indicate fundamental resonance frequencies overlapping for two hits within $\pm 1 \mathrm{kHz}$; bold dashed vertical lines indicate fundamental frequencies overlapping for more than 2 hits within $\pm 1 \mathrm{kHz}$. The change in the fundamental fre-

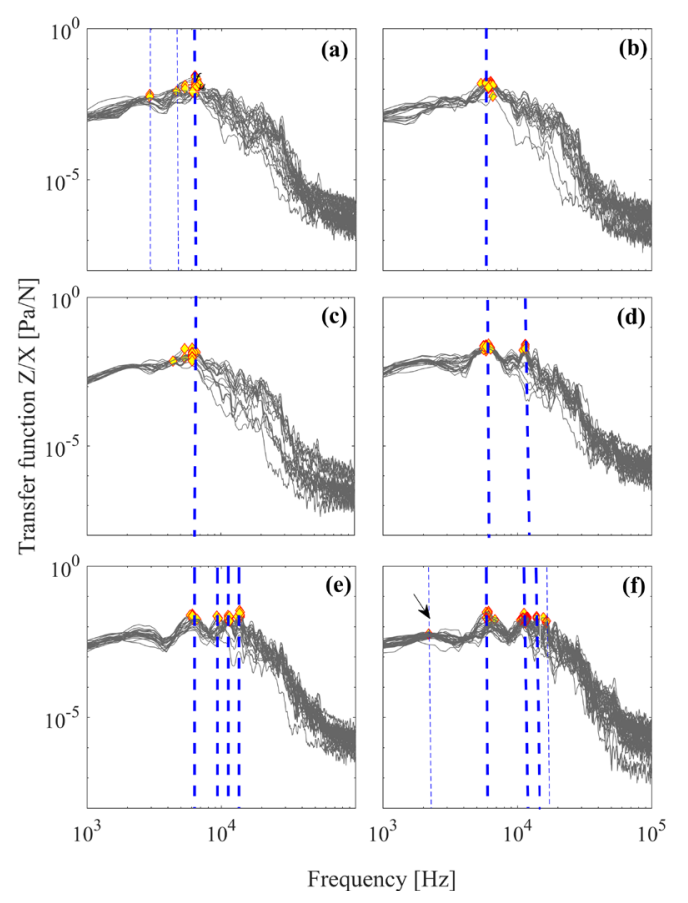

Figure 5. Vibroacoustic transfer functions of all six broaches: For broaches 1 to 3 compared to broaches 4 to 6 the transfer function does not change its fundamental frequency very much as indicated by the smaller number dashed lines.

quency with each impact does not show a trend. However, with increasing broach size, the fundamental frequency initially found at about $6.1 \mathrm{kHz}$ changes its position more, with a tendency to move towards higher frequencies with the spectra becoming more broadband. Similar to [11], also a low frequency resonance is picked up (indicated by arrow in Fig. 5(f)): whether this is due to a standing wave 
within the bone [11] or due to other effects cannot be determined.

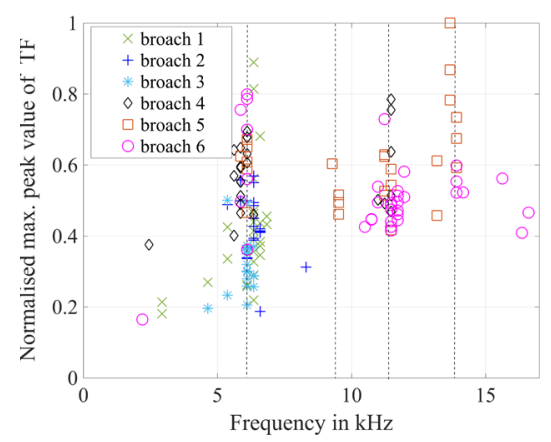

Figure 6. Normalised maximum peak values of the fundamental frequency of the transfer function over resonance frequencies

Next, the peak values as picked from the transfer function are normalised relative to their maximum absolute value and plotted against their frequencies in Fig. 6. Four clusters become visible: around $6.10 \mathrm{kHz}$ (the most often occurring), $9.28 \mathrm{kHz}$ (only for broach 5), $11.47 \mathrm{kHz}$ and $13.92 \mathrm{kHz}$ (indicated by vertical dashed lines).

\subsection{Nonlinear time series analysis}

Fig. 7(a) shows the real-valued impulse response $g(t)$ and Figs. 7(b) and (c) exemplify the extraction of the delay and a suitable embedding dimension[23, 25].
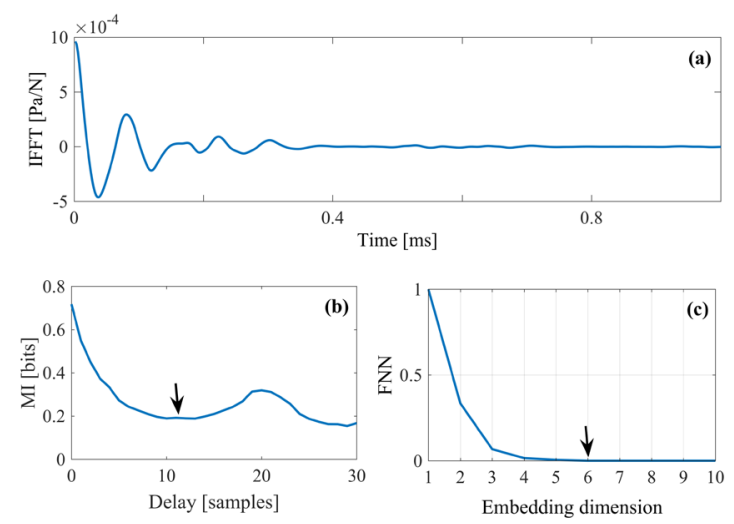

Figure 7. Steps of estimating the delay and the dimension (indicated by arrows) using the (a) impulse response: (b) mutual information, and (c) false nearest neighbour algorithm.

The delay and the embedding dimension for each broach are grouped and analysed using one-way ANOVA tests. The results for the delay and the embedding dimension showed significant differences between the broaches $(S S=845.83, d f=148, M S=78.15, F=24.56$, $p<<0.001)$ and $(S S=169.05, d f=148, M S=8.71$, $F=9.92, p<<0.001)$. Results of the posthoc analysis with BONFERRONI correction are depicted in Fig. 8 together with its $95 \%$ confidence intervals (lines). The larger the broach, the smaller the embedding delay and the larger the embedding dimension. Two groups are formed with significantly different delays and dimensions since their $95 \%$ confidence intervals do not overlap; only broach 4 is not significantly different, to either group.

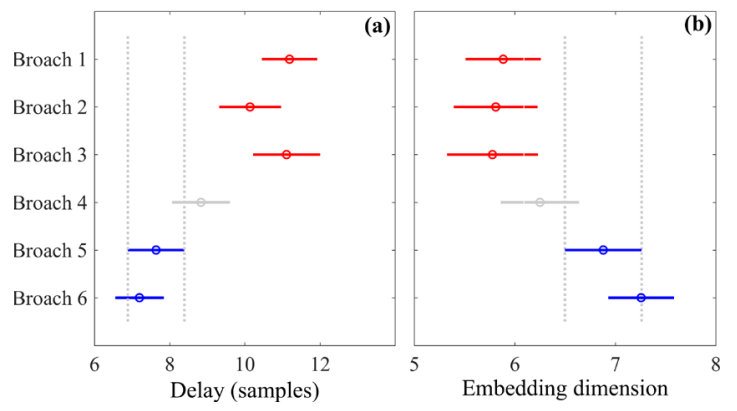

Figure 8. Results of the statistical analysis multi-compare posthoc test: average (a) delay, and (b) embedding dimension with $95 \%$ confidence interval.

\section{Discussion}

In this cadaver study we simulated the procedure of a total hip arthroplasty using compaction broaches. By analysing qualitatively the relationship between impaction forces and their resulting sound pressure, we identified at least two groups of broaches with different behaviours as indicated by a superposed GAUSSIAN distribution (multimodal) and an increase of heteroscedasticity which cannot be explained with the statistical analysis alone. In particular the fourth broach showed relative loud impaction sounds.

The acoustic transfer functions obtained here show that the acoustic radiation of the tissue/bone-broach interface of the cadaver-implant-system depends on the size of the broaches and the working progress during impaction. With increasing broach size the magnitude at the fundamental frequency seems to show a tendency to increase; especially the last three broaches show an up to 5 times higher ability to radiate sound per unit impaction force; however considering their magnitudes the difference would be generally only below $2 \mathrm{~dB}$. With increasing working progress the data also seems to scatter more (heteroscedasticity).

Whitwell et al. [11] observed a shift of the fundamental frequency to a lower frequency and assumed that this is due to a standing wave within the bone once the ideal cavity is prepared. However, we found that as a result of the cavity preparation impacting increasing broach sizes, the system's fundamental frequency changed its location and started fluctuating around the system's initial fundamental frequency of around $6.1 \mathrm{kHz}$ with a tendency towards higher frequencies and also to become more broadband.

This qualitative change in the complexity of the system was consequently quantified by calculating complexity measures [25] such as the averaged auto-mutual information and the false nearest neighbour algorithm from the impulse response function to obtain the delay and the global embedding dimension respectively [25]. As the broach size increased, the delay time decreased but the embedding 
dimension increased, indicating a decreased predictability of the system and an increased complexity of the impulse response function. More complex dynamics require a greater phase space dimension in order to describe the system's dynamics [23]. Whether this increased complexity is due to the broach positioning (better coupling between bone and broach) or micro-fractures (hence an indication to stop the impaction process [12]) needs to be studied further e.g. by ultrasound for damage detection.

Results of this study encourage the development of an instrumented hammer to use nonlinear analysis tools based on complexity measures in a data stream. This hammer could serve the surgeon alone or in conjunction with a robotic arm as a diagnostic tool during the implantation procedure and enhance implant positioning. Similar devices could be applied for knee and shoulder prostheses. The development of such a technology would reduce the likelihood of revision surgeries (currently at about $9.6 \%$ [2]), would have a major impact on patients recovery times and well-being, and reduce the overall treatment costs of total arthroplasties.

The determination of a proper time lag and embedding dimension, however, can be problematic. Here the ideal numbers of neighbours to be searched for is difficult to determine. While the mutual information and the false nearest neighbourhood algorithm are well-established and mature methods, more sophisticated e.g. adaptive neighbourhood search algorithms [26] could enhance the performance in cases of varying measurement quality. Owing to the rather good quality of our data, we did not encounter problems during the nonlinear time series analysis step and the TIKHONOV regularisation performed well. However, during total hip arthroplasties operations, employing an instrumented hammer, signal processing in a data stream and optimised algorithms for real-time processing capability would be desirable.

\section{Declarations and Acknowledgements}

The authors declare that no conflict of interest exists and the study was approved by the Ethics Commission of the Medical Association Hamburg. SO acknowledges the receipt of the Australia Award/ Endeavour Research Fellowship (ERF PDR 5563 2016) awarded by the Australian Government.

\section{References}

[1] A. Carr, O. Robertsson, S. Graves, A. Price, N. Arden, A. Judge, B. D.J., The Lancet 379, 1331 (2012)

[2] N.J.R. Registry, Tech. rep., Australian Orthopaedic Association (2016)

[3] S. Oberst, Z. Zhang, G. Campbell, M. Morlock, J. Lai, N. Hoffmann, Towards the understanding of hip squeak in total hip arthroplasty using analytical contact models with uncertainty, in Proceedings of the INTER-NOISE 2016 - 45th International Congress and Exposition on Noise Control Engineering: Towards a Quieter Future 21-24 August 2016, Pages 5539-5549 (2016)
[4] E. Askari, P. Flores, D. Dabirrahmani, R. Appleyard, Tribology International 93, 239 (2016)

[5] K. Sø balle, E.S. Hansen, H. B.-Rasmussen, P.H. Jø rgensen, C. Bü nger, Journal of Orthopaedic Research 10, 285 (1992)

[6] J. Bätz, K. Püschel, A. Klein, M. Morlock, G. Campbell, Determination of bone-cavity interface densification after femoral cavity broaching using QCT, in 22nd Congress of the European Society of Biomechanics, July 10 - 13, 2016, Lyon, France (1997), p. 1

[7] R. Sakai, A. Kikuchi, T. Morita, N. Takahira, K. Uchiyama, T. Yamamoto, M. Moriya, K. Uchida, K. Fukushima, K. Tanaka et al., Hip International 21, 718 (2011)

[8] D. Romness, D. Lewallen, Bone \& Joint Journal 72B, 761 (1990)

[9] N. Yagi, Y. Oshiro, O. Ishikawa, Y. Hata, N. Shibanuma, 2011 IEEE Workshop on Robotic Intelligence In Informationally Structured Space 12133871, 32 (2011)

[10] V. Mathieu, A. Michel, C. Flouzat Lachaniette, A. Poignard, P. Hernigou, J. Allain, G. Ha iat, Medical Engineering \& Physics 35, 1558 (2013)

[11] G. Whitwell, C. Brockett, S. Young, M. Stone, T. Stewart, Proceedings of the Institution of Mechanical Engineers. Part H, Journal of Engineering in Medicine 227, 175 (2012)

[12] I. Morohashi, H. Iwase, A. Kanda, T. Sato, Y. Homma, A. Mogami, O. Obayashi, K. Kaneko, SICOT J 2017, 3, 13 3, 1 (2017)

[13] N.H. Fletcher, T.D. Rossing, The physics of musical instruments (Springer-Verlag New York, 1998)

[14] E. Askari, P. Flores, D. Dabirrahmani, R. Appleyard, Nonlinear Dynamics 82, 1039 (2015)

[15] S. Oberst, J.C.S. Lai, Journal of Sound and Vibration 330, 2978 (2011)

[16] J. DiSteffano, A. Stubberud, I. Williams, Schaum's Outline of Feedback and Control Systems, Second Edition (McGraw-Hill Education: New York, Chicago, San Francisco, Athens, London, Madrid, Mexico City, Milan, New Delhi, Singapore, Sydney, Toronto, 2014)

[17] E. Oberst, S. Nava-Baro, J.C.S. Lai, T.A. Evans, Acoustics Australia 43(1), 87 (2015)

[18] D. Gubbins, Time Series Analysis and Inverse Theory for Geophysicists (Cambridge University Press, 2004)

[19] S. Grace Chang, B. Yu, M. Vetterli, IEEE Transactions on image processing 9, 1532 (2000)

[20] A. Tikhonov, V.Y. Arsenin, F. John, Solutions of illposed problems (John Wiley \& Sons, New York, Toronto, London, Sydney, 1977)

[21] A.M. Fraser, H.L. Swinney, Physical Review A 33, 1134 (1986)

[22] D. Abásolo, J. Escudero, R. Hornero, C. Gómez, P. Espino, Medical \& Biological Engineering \& Computing 46, 1019 (2008) 
[23] H.D.I. Abarbanel, Analysis of observed chaotic data (Springer: New York, 1996)

[24] N. Marwan, M. Carmen Romano, M. Thiel, J. Kurths, Physics Reports 438, 237 (2007)

[25] S. Oberst, J. Lai, Journal of Sound and Vibration 334, $120(2015)$
[26] N. Garcia-Pedrajas, J. A. Romero del Castillo, G. Gonzalo Cerruela-Garcia, IEEE Transactions on Neural Networks and Learning Systems pp. 470-475 (2015) 